\section{Drogeninduziertes Lernen: Sensitivierung bei Apomorphin}

\author{
Juan D. Delius, Martin J. Acerbo, Sabine Keller und Adriana Godoy
}

\section{Zusammenfassung}

Der Verhaltenseffekt der Psychostimulanzien Kokain und Amphetamin, vornehmlich indirekte Dopaminagonisten, steigert sich bei wiederholter Einnahme ein und derselben Dosis. Wahrscheinlich spielt diese Sensitivierung bei der Suchtentstehung eine wichtige Rolle. Mehrfache Gaben vom direkten, nicht suchtbildenden Dopaminagonist Apomorphin (Apo) führen ebenfalls zu einer Sensitivierung. Wir berichten, dass diese Empfindlichkeitssteigerung besonders gut an den Pickanfällen, die Apo bei Vögeln auslöst, untersucht werden kann. Es werden Versuchsergebnisse vorgestellt, die zeigen, dass die Sensitivierung auf einen langwährenden Pawlovschen Lernvorgang zurückgeht. Die Sensitivierung ist ausgesprochen umgetungsspezifisch, auch hinsichtlich der Pickziele. Einige Besonderheiten der Sensitivierung erklären sich dadurch, dass Apo ein unkonditionierter Reiz ist, der gleichzeitig auch Eigenschaften eines konditionierten Reizes hat. Neuropharmakologische Ergebnisse suggerieren, dass die Sensitivierung auf Apo auf eine synaptische Interaktion zurückgeht, die zu einer Effektivitätssteigerung der glutamatergen Übertragung im ventralen Striatum führt.

\begin{abstract}
Drug-induced learning: Sensitization to apomorphine.

The effect on behaviour of psychostimulants such as cocaine and amphetamine, mainly indirect dopamine agonists, increases with repeated consumption of one and the same dosage. This sensitization probably plays an important role during the development of addiction. Repeated administration of apomorphin (apo), a direct, non-addictive dopamine agonist also leads to sensitization. Here we report that this increased reactivity can be advantageously studied using the pecking fits that apo causes in birds. Experimental results are presented which show that the sensitization is based on a lasting pavlovian learning process. The sensitization is strongly related to specific contexts, even concerning the pecking targets. Some peculiarities of the sensitization can be accounted by the fact that besides being an unconditioned stimulus apo also has conditioned stimulus properties. Neuropharmacological results suggest that the sensitization to apo is based on a synaptic interaction leading to a glutamatergic transmission efficacy increase in the ventral striatum.
\end{abstract}

Keywords: psychostimulants, sensitization, addiction, apomorphine, classical conditioning

\section{Einleitung}

Es ist funktionell nachvollziehbar, dass es auf wiederholte Gabe oder Einnahme eines Neurotransmitters/modulators bzw. seiner Agonisten zu einer regulierenden Empfindlichkeitsminderung, also Toleranz kommt. Gleichermaßen ist es einsichtig, wieso das dann zu einer kompensierenden, süchtigen Mehreinnahme einer Droge (z. B. Morphium) führen kann. Ebenfalls homeostatisch naheliegend ist es, wenn es bei fortgesetzter Gabe eines Transmitterantagonisten (z. B. Haloperidol, Hal) zu einer Empfindlichkeitssteigerung, also Sensitivierung auf den
Transmitter (im Beispiel auf Dopamin, Da) und seine Agonisten kommt. Weniger verständlich ist aber, warum wiederholte Gaben von Psychostimulanzien (z. B. Amphetamin, Kokain, beides vornehmlich Da-Agonisten) zu einer Empfindlichkeitssteigerung führen. Wie auch immer, neben einer Sensitivierung bezüglich der psychomotorischen Effekte kommt es wohl auch zu einer Steigerung des Anreizwertes der Drogen, was zu einem suchtartigen Verlangen nach ihnen führt (Robinson und Berridge 1993). Dieser Umstand hat zu einer umfang- und ergebnisreichen Forschung zur Sensitivierung bei Psychostimulanzien geführt (Anagnostaras und Robinson 1996). Sie ist aber auch reich an Widersprüchen, unserer Meinung nach vielleicht, weil Amphetamin und Kokain indirekt und unspezifisch Da-Agonisten sind.

Wir haben uns statt dessen Apomorphin (Apo), einem synthetischen Abkömmling von Morphium, zugewandt (Abbildung 1A). Apo ist ein sehr spezifischer und effektiver Da-Agonist, der in seiner Wirksamkeit den Transmitter selbst übertrifft. Apo bindet sowohl an die Da-Rezeptoren der Adenylatzyklase anregenden D1-Familie als auch an diejenigen der Adenylatzyklase hemmenden D2-Familie. Dass Apo über präsynaptische Rezeptoren die Da-Ausschüttung hemmt, spielt im hiesigen Kontext wegen der mehr als kompensierenden postsynaptischen Wirkung keine Rolle. Auf den präsynaptischen Da-Transporter hat es wohl keinen Einfluss. Apo wird übrigens verhältnismäßig schnell wieder abgebaut bzw. ausgeschieden: es hat eine Halbwertzeit von etwa einer halben Stunde. Wieso aber Apo, das sich strukturell von $\mathrm{Da}$ doch sehr unterscheidet, sich an die Da-Rezeptoren so besonders effizient bindet, ist bisher noch nicht hinreichend geklärt. Die Vermutung, dass eine opioide Nebenwirkung mitbeteiligt sein könnte, ist unwahrscheinlich, da der Opiatantagonist Naloxon die Wirksamkeit von Apo nicht beeinträchtigt. Klinisch wird es seit langem als Emetikum (hohe Dosen) und neuerdings als Erektivum (niedrige Dosen) genutzt. Gelegentlich wird es zur Behandlung von akuten Parkinsonsymptomen eingesetzt. Daneben bewirkt es eine erhöhte Somatostatin- und eine verminderte Prolaktinausschüttung und eine Hypothermie mit paradoxen Hitzeempfindungen. Bekannt ist aber auch, dass Apo bei Nagern, ähnlich wie Kokain und Amphetamin, motorische Hyperaktivität induziert, die bei geeigneter Dosierung von stereotypen oralen Reaktionen (Schnüffeln, Beißen, Nagen, Kauen) unterbrochen wird. Tatsächlich löst Apo bei Schnecken, Fischen, Amphibien, Reptilien, Vögeln und Säugern solcherart orale Stereotypien aus. Bei Vögeln, insbesondere Tauben und Hühner, führen etwa 0,5 mg Apo per kg Körpergewicht, wenn erstmals i.m. gegeben, zu einem regelrechten Pickanfall, der aus mehreren Hundert Pickern besteht und etwa eine Stunde dauert (Brunelli et al. 1975; Machlis 1980). Lokomotionsaktivität ist bei den Vögeln wenig ausgeprägt und interferiert kaum mit dem Picken. Weniger dominant sind auch Gefiederputzbewegungen (bei Nagern besonders Gesichtwaschen), Strecken und Gähnen, die meist bei niedrigeren Dosen Apo auftreten (Delius 1988). Menschen üb- 
rigens zeigen nach Apoeinnahmen u.U. auch Kau- und Gähnstereotypien (Lal 1988; Szechtman 1987). Anders als Kokain und Amphetamin ist es bei ihnen nicht suchtauslösend und das, obwohl gezeigt wurde, dass Ratten und Affen durchaus bereit sind, sich fortlaufend per Hebelschalter kleine Dosen Apo intravenös zu spritzen. Hier aber beschäftigt uns das vergleichsweise sehr apospezifische Picken der Tauben, das wir der viel untersuchten aber viel weniger apospezifischen Laufaktivität von Mäusen und Ratten vorgezogen haben.

Pickhandlungen werden von Tauben in verschiedensten Verhaltenskontexten eingesetzt, so z.B. bei Aggression und Nestbau, aber natürlich vorwiegend beim Fressen. Nur sehr ausgehungerte Tauben, die aus einem duelle Idiosynkrasien aus. Das Ziel, das die einzelnen Tauben für ihre ersten paar Picker unter Apo eher zufällig wählen, ist stark bestimmend.

Bei der Standarddosis von $0,5 \mathrm{mg} / \mathrm{kg}$ Apo und in einem mit vielen Farbpunkten als Pickziel versehenen Käfig beginnt das Pikken wenige Minuten nach der Injektion, erreicht nach etwa 15 min ein Maximum von rund 100 Pickern/min und geht nach etwas mehr als einer Stunde nach der Injektion gegen Null. Apodosen zwischen 0,2 und 2 $\mathrm{mg} / \mathrm{kg}$ führen zu schwachen bis starken Pickanfällen, wobei sich die Latenz des Pickbeginns verkürzt und die maximale Pickfrequenz sowie die Pickdauer erhöhen. Hohe Dosen bis etwa $5 \mathrm{mg} / \mathrm{kg}$ rufen zwar auch noch Picken hervor, führen aber auch zu
A
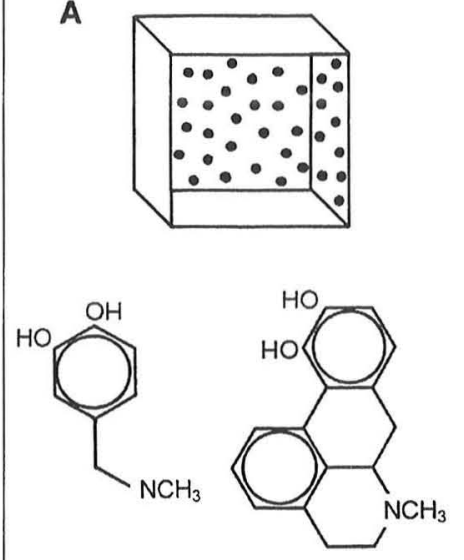

Dopamin

Apomorphin

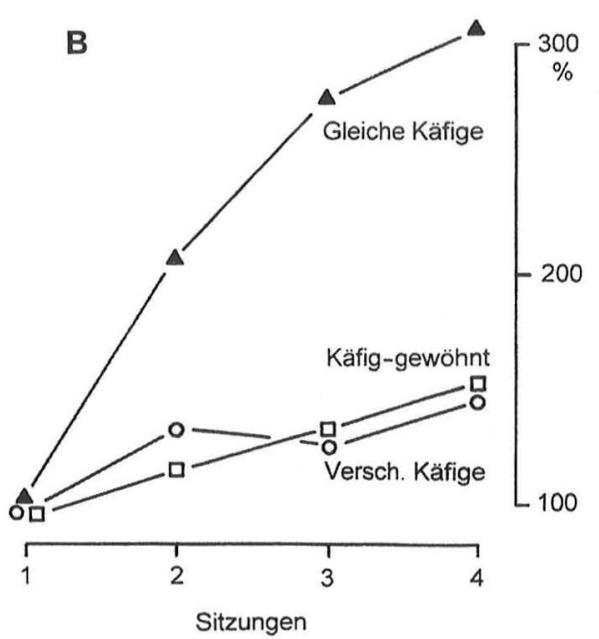

Sitzungen

Abb. 1: A: Dopamin, Apomorphin und typischer Versuchskäfig. B: Umgebungsabhängige Sensitivierung (verändert nach Keller et al. 2002).

Kiesgemisch Körner herauslesen oder dressiert wurden, einen Schalter für gelegentliche Körnerbelohnungen zu picken, zeigen einen Pickaktivitätsschub, der dem apoinduzierten in etwa vergleichbar ist. Das Apopicken ähnelt sehr dem, welches bei der Futterbeschaffung beobachtet wird. Gelegentlich richten die Tauben diese Picker auf Körner, aber lassen sie meist wieder fallen. Obwohl das apoverursachte Picken durch Futterdeprivation gefördert wird, hat die Droge einen anorektischen Nebeneffekt (Deviche 1984). In der Regel picken apobehandelte Tauben nicht-nahrhafte, kleinere, kontrastreiche Reize wie z.B. Nietenköpfe, Schweißpunkte, Farbpunkte auf dem Käfigboden oder den Käfigwänden. Gelegentlich picken apobehandelte Tauben ihre eigenen Zehen oder Federn, wobei letzteres zum o.g. Gefiederputzen übergehen kann. Betreffend dieser Ziele bilden sich sehr stabile indivi-
Würgen und Schwanken, was mit dem Pikken interferiert. Bei niedrigeren Dosen bis etwa $0,05 \mathrm{mg} / \mathrm{kg}$ tritt eher regelrechtes Gefiederputzen auf. Die gelegentlichen Picker sind dann auf sogenannte Intentionsbewegungen reduziert und bestehen lediglich aus einer mit Augenkonvergenz verbundenen Fixierung eines potentiellen Pickzieles. Übrigens, dass das Picken auf Apo hin von einer gleichzeitigen Aktivierung von D1-Typund D2-Typ-Rezeptoren abhängt, ist mittels der Koadministration von spezifischen D1und D2-Antagonisten festgestellt worden (s.u.; Zarrindast et al. 1992). Warum es bisher nicht gelungen ist, mit Kombinationen von entsprechenden Agonisten (z. B. mit SKF38393 und Quinpirol) kaum mehr als Pickreaktionsandeutungen hervorzurufen, bleibt aber unklar. Kokain und Amphetamin sind übrigens nur sehr schwach pickauslösend.
Es gibt aber vereinzelt Tauben, die auch bei höheren Dosen von Apo kaum eine Pickreaktion zeigen. Züchtungen haben erwiesen, dass diese mangelnde Reaktivität einen genetischen Hintergrund hat. Die Tiere scheinen aber bezüglich ihres übrigen Verhaltens, einschließlich Futterpicken und Unterscheidungslernen, völlig normal zu sein. Möglicherweise werden diese Mutanten uns helfen, Einblicke in die genauere Wirkungsweise von Apo zu gewinnen.

\section{Apomorphin-Sensitivierung}

Früh fiel uns auf, dass sich bei wiederholten Gaben einer bestimmten Dosis Apo die Intensität der Pickanfälle, die sie auslösten, steigerte. Erst nach etwa 5 bis 6 täglichen Injektionen von z.B. unserer Standarddosis $0,5 \mathrm{mg} / \mathrm{kg}$ Apo stellt sich eine Reaktionsasymptote ein. Auf die erste Apoinjektion hin zeigen die Tauben mehrere Hunderte Picker. Asymptotisch löst die gleiche Dosis mehrere Tausend Picker aus (Delius 1985, Wynne und Delius 1995; siehe auch Abbildungen 2A und 4A). Diesen Reaktionszuwachs nennt man Sensitivierung. Die Reaktionsasymptoten, die sich einstellen, sind dosisabhängig, aber der Anstieg ist nur schwach dosisbestimmt. Ab etwa 1 $\mathrm{mg} / \mathrm{kg}$ Apo kommt es allerdings zu einer Sättigung der Asymptoten, weil die Tiere dann so schnell picken, wie physisch überhaupt möglich (etwa 180 Picker/min). Wenn eine Gesamtdosis von $1,8 \mathrm{mg} / \mathrm{kg}$ Apo mittels einer implantierten Osmopumpe kontinuierlich über 6 Tage injiziert wurde, kam es zu keinem nennenswerten Picken. Anschließend war auch keine sensitivierte Reaktion auf eine $0,5 \mathrm{mg} / \mathrm{kg}$ Dosis festzustellen, wie das nach einer täglichen Dosis von $0,3 \mathrm{mg} / \mathrm{kg}$ über dieselbe Zeit eingetreten wäre. Darüber hinaus zeigen die infusionvorbehandelten Tiere eine deutlich verminderte Sensitivierung auf eine fortgesetzte tägliche Verabreichung der Standarddosis. Beides lässt vermuten, dass es bei einem stetigem Apospiegel zu einer gewissen Toleranz gegenüber der Droge kommt. Bei einer intermittierenden Apoverabreichung wirken sich zeitliche Abstände von 3 Stunden bis 5 Tage zwischen den einzelnen Injektionen nur geringfügig auf den Verlauf der Sensitivierung aus: kürzere Intervalle führen sogar zu einer leicht höheren Pickasymptote als längere. Wegen der kurzen Verbleibezeit von Apo (s.o.) dürfte eine Akkumulation der Droge im Körper dabei kaum eine Rolle spielen.

Wichtig ist, dass - wenn einmal zustandegekommen - die Sensitivierung dann 
ausgesprochen dauerhaft ist. Erst nach etwa zwei Jahren fällt die sensitivierte Pickreaktion, welche die Standarddosis Apo auslöste, nur auf die Hälfte zurück. Allgemeiner muss man damit rechnen, dass eine spezifische Vorgeschichte mit Apo einen deutlichen Einfluss auf die Wirksamkeit späterer Apoapplikationen haben. Godoy und Delius (1999) fanden, dass die Reihenfolge, mit der eine $0,2 \mathrm{mg} / \mathrm{kg}$ Dosis und die Standarddosis jeweils über mehrere Tage angewendet wurde, $\mathrm{zu}$ verschiedenen Reaktionsasymptoten für ein und dieselbe Dosis führte. Nach einer Standarddosis Vorbehandlung führte eine $0,2 \mathrm{mg} / \mathrm{kg}$ Dosis zu einer erhöhten Reaktion und nach einer 0,2 Vorbehandlung führte die Standarddosis zu einer verminderten Reaktion. Es ergab sich tatsächlich eine weitgehende Angleichung der Effekte beider Dosierungen, die sonst natürlich deutlich unterschiedlich wirken (s.o.).

Es fiel uns bald auf, dass nach einer Sensitivierungsbehandlung die Tauben auch dann, wenn sie unbehandelt oder mit Saline (Sal) gespritzt in den Versuchskäfig gesetzt wurden, geringere aber dennoch deutliche Pickschübe zeigten. Lindenblatt und Delius (1987) führten daraufhin einen Differenzierungsversuch nach Pawlov durch. Zwei unterschiedlich dekorierte Versuchskäfige (gelbe Punkte/schwarzer Hintergrund, grüne Punkte/weißer Hintergrund) wurden verwendet. Sie stellten zwei verschiedene konditionierende Stimuli (CS) dar. Tauben wurden wiederholt in den einen Käfig (der CS+) gesetzt, nachdem sie mit Apo injiziert worden waren (der unkonditionierte Stimulus US) und zeigten dort das Apo-induzierte Picken (die unkonditionierte Reaktion, UR). Abwechselnd wurden sie mit Sal behandelt und in den anderen Käfig (der CS-) gesetzt, wo sie natürlich so gut wie nicht pickten. Bei anschlieBenden Tests wurden sie nur noch nach Salinjektionen, sowohl in den einen, als auch in den anderen Versuchskäfig gesetzt. Während die Tauben im vormals salkontingenten CS- Käfig nur einige wenige Picker produzierten, zeigten sie im vormals apokontingentem CS+ Käfig etliche Dutzend Pikker. Das zeigte, dass letztere Reaktion eine konditionierte Reaktion (CR) war. Wynne und Delius (1995) führten zur Bestätigung einen weiteren Versuch durch, bei dem aber eine Latenzverkürzung der Pickreaktion, die durch das Hinzukommen des schnelleren, käfigbedingten CR zur langsameren, drogenbedingten UR zustande kam, im Vordergrund stand. Sie zeigten, dass sich während einer differentiellen Konditionierung der Pickschubbeginn (definiert als Dauer bis zum jeweils fünften Picker der Sitzungen) nach einer Standarddosis Apo und unmittelbar anschließender Einführung in den CS+ Käfig sich tatsächlich von anfänglich etwa 12 min auf etwa 3 min verkürzte. Bei anschließenden Tests, bei denen die Tauben nach Gaben einer geringeren 0,2 mg/ kg Apo Dosis sowohl in den CS- als auch in den CS+ Käfig gesetzt wurden, führte ersterer zu einer Picklatenz von ca. $14 \mathrm{~min}$, aber letzterer zu einer von nur rund $3 \mathrm{~min}$.

Godoy und Delius (1999) gingen einen Schritt weiter und zeigten, dass der Reaktionszuwachs, der der Sensitivierung auf Apo zugrundeliegt, streng kontextabhängig ist, d.h. dass er nur, oder zumindest beinahe nur, in dem Versuchskäfig auftritt, in dem die Tauben bei der Sensitivierungsbehandlung gleich nach den Apoinjektionen ihren Pickanfall erlebt haben. Dazu testeten sie Tauben nach Apogabe, entweder in dem gleichen oder einem unterschiedlichen Käfig als dem, der zur Sensitivierungsbehandlung benutzt wurde. War der Testkäfig wirklich gründlich verschieden von dem Trainingskäfig (z.B. zylindrisch anstatt kubisch), dann zeigte sich die Sensitivierung nicht in dem Testkäfig, obwohl sie sich natürlich voll im Trainingskäfig auswirkte. Das Pickverhalten von geeignet kontrollbehandelten Tauben stellte sicher, dass das geringere Picken im abweichenden Käfig nicht auf dessen Ungewohntheit, also nicht auf ein Fremdeln der Tauben, zurückführbar war. Übrigens, für die Entwicklung der käfigspezifischen Sensitivierung sind nach den jeweiligen Apoverabreichungen nur die ersten $20 \mathrm{~min}$ Aufenthalt im maßgeblichen Versuchskäfig ausschlaggebend. Die weiteren 40 min, die der Pickanfall dauert, veranlassen keine zusätzliche Konditionierung. Der neuronal-molekulare Lernprozess, der durch Apo angestoßen wird, ist somit nach 20 min gesättigt (Keller et al. 2002).

Weil allgemeiner das Vorherrschen kontextab- oder kontextunabhängiger Sensitivierungen bei Psychostimulanzien kontrovers diskutiert wird, wurde hierzu ein weiterer Versuch gemacht. Drei Taubengruppen wurden einer viertägigen Sensitivierungsbehandlung unterzogen, wobei die Tauben der ,verschieden "Gruppe jeweils nacheinander in vier deutlich unterschiedlichen Käfigen einen Apopickanfall erlebten. Die Tauben der ,gewöhnten“ Gruppe wurden auch so behandelt, lernten aber ge- trennt auch ihren letzten Apoversuchskäfig vorher an drei Tagen nach Salinjektionen kennen. Die Tauben der ,gleichen“ Gruppe erlebten immer im selben Käfig ihren Apopickanfall. Es stellte sich heraus, dass nur die ,gleiche“ Gruppe einen sehr bedeutsamen Reaktionszuwachs zeigte, während die beiden anderen Gruppen nur eine geringere und untereinander nicht unterschiedliche Sensitivierung aufwiesen. Diese Restsensitivierung dürfte $\mathrm{z}$. T. auf die gleichbleibende Käfigvorderaussicht und auf den Drogenzustand (s.u.) zurückgehen. Hiermit wurde nochmalig die ausgesprochene Umgebungsabhängigkeit der Sensitivierung auf Apo und die unbedeutende Rolle der simplen Bekanntheit dieser Umgebung belegt (Abbildung 1B).

Von Reizen, die im Zusammenhang mit Konditionierungsversuchen die Eigenschaften eines UR aufweisen, erwartet man meist, dass sie auch eine verstärkende, hier belohnende Wirkung haben. Um dies zu prüfen, führten Burg et al. (1989) einen sogenannten Ortsbevorzugungsversuch durch. Tau-

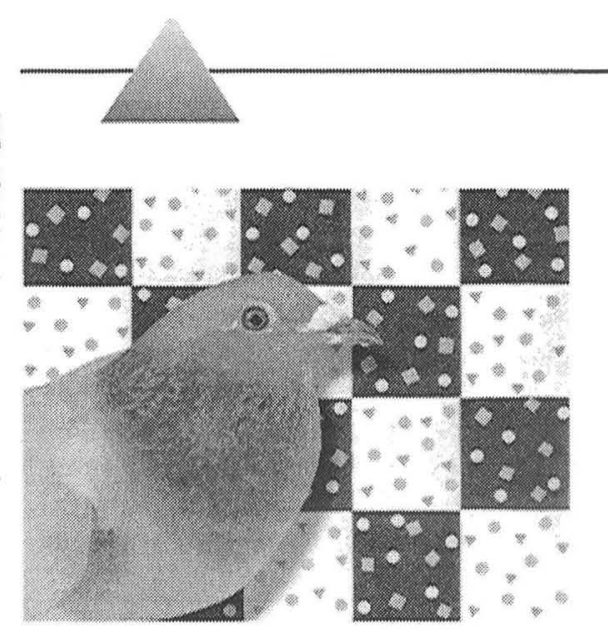

Zum Titelbild:

Apomorphin behandelte Tauben picken eifrigst Farbpunkte und lernen dabei sehr wählerisch zu sein. Bild: AVM-Foto, Uni Konstanz 
ben verblieben zunächst wiederholt und abwechselnd in zwei verschiedenen Zielkäfigen einer T-förmigen Wahlapparatur und zwar so, dass sie den einen immer unter dem Einfluss von Apo und den anderen unter dem Einfluss von Sal erlebten. Nach Abschluss dieses Trainings wurden die Tiere salgespritzt. Sie wurden mehrmals in den Startgang der Wahlapparatur gesetzt und durften zwischen den beiden Zielkäfigen wählen. Sie wählten bei mehr als $75 \%$ der Tests, also deutlich überzufällig, den Käfig, den sie zuvor unter Apoeinfluss erlebt hatten. Die Bevorzugung des Drogenkäfigs zeigt an, dass Apo bei Tauben eine appetitive, belohnende Wirkung hatte. Dies steht damit im Einklang, dass das belohnungssignalisierende tegmento-telencephale Projektionssystem, über welches Tauben genauso wie Säuger verfügen, sich wesentlich des Transmitters Da bedient (Delius und Pellander 1982, Gardner 1997).

\section{Konditionierte Sensitivierung?}

Die bisher referierten Ergebnisse legen nahe, dass der Pickreaktionszuwachs bei der Sensitivierung auf Apo durch Konditionierung zustandekommt, d.h. dadurch, dass die zunehmende, durch die Käfigumgebung hervorgerufene CR sich auf die durch Apo ausgelöste UR hinzuaddiert (CR+UR). Nicht übereinstimmend hiermit ist allerdings die bisher beiläufig erwähnte Tatsache, dass die CR, die der apokontingente CS Käfig bei einer Salbehandlung auslöst, bestenfalls einige Hundert Picker beträgt, während der Sensitivierungszuwachs, also die in der o.g. Additionsformel implizierte CR, einige Tautionierte Pickreaktion nach Sal sich mittels einer Übersensitivierung - längere Fortsetzung der Apobehandlung nach Erreichen der Reaktionsasymptote - steigern lässt, bleibt send Picker beträgt. Auch wenn die kondi-

sie immer noch weit unter dem Sensitivierungszuwachs.

Ebenfalls mit der Konditionierungshypothese ist nicht passend, dass ein mehrfach wiederholtes Einsetzen in den CS Käfig nach Sal zwar zur Verminderung (Löschung) des dazugehörigen CR führt, aber gar nicht oder nur geringfügig (Abbildung 2A; Delius et al., in Vorb.) die sensitivierte Apopickreaktion mindert. Ähnlich unpassend ist, dass eine ausgedehnte Vorabgewöhnung an den Versuchskäfig anschließend nicht zu einer geminderten Aposensitivierung führt. Üblicherweise haben solche CS - kein US und Nach- und Vorbehandlungen bei der Pawlovschen Konditionierung eine ausgesprochen hemmende Wirkung.

Im Rahmen der Konditionierungshypothese ist es aber sicher naiv, die Gabe von Apo mit einem üblichen US gleichzusetzen. Systemisches Apo dürfte eine verzweigtere Wirkung als z.B. Futtergaben haben. Da Rezeptoren nachgewiesenermaßen auch in sensorischen Systemen (z.B. Retina und Tectum) vorkommen, muss man vermuten, dass Apogaben - wie menschliche Probanden auch tatsächlich angeben - zu einer wahrnehmbaren Zustandsänderung führt. Bei Tauben haben wir mittels eines Drogenunterscheidungsexperiments genau dies auch zeigen können. Hungrig gehaltene Tauben lernten, um sich automatisch Futter zu besorgen, von zwei Pickschaltern verlässlich, wenn sie vorher mit $0,25 \mathrm{mg} / \mathrm{kg}$ Apo gespritzt wurden, den roten, linken zu wählen oder aber den grünen, rechten, wenn sie vorher mit Sal gespritzt wurden. Sie konnten also offensichtlich verhaltenswirksam empfinden, ob sie unter Apoeinfluss standen oder nicht. Diesen Apozustand schienen sie übrigens nicht mit einem Kokain- oder Amphetaminzustand $\mathrm{zu}$ verwechseln (Abbildung 2B).

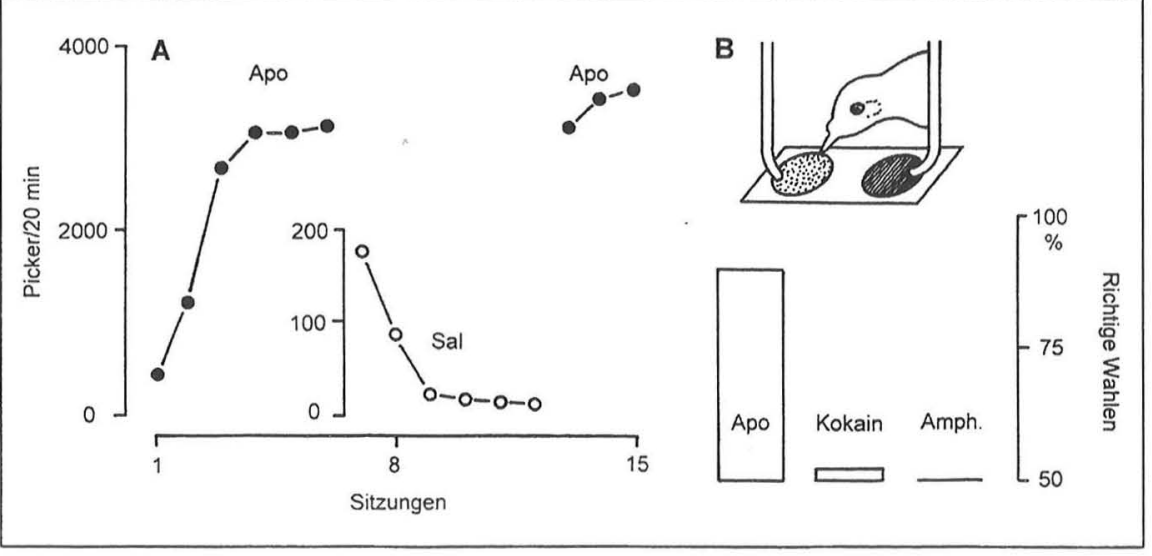

Abb. 2.: A: Fehlender Extinktionseffekt. B: Drogenzustandsdiskrimination (verändert nach Godoy und Delius 1999, Delius et al., in Vorb.).
Außer dem exterozeptiven CS Versuchskäfig gäbe es demnach auch einen interozeptiven mit CS Aponebenwirkung. Dieser Umstand sollte zu einer sogen. Selbstkonditionierung auf Apo führen. Um dies zu prüfen, haben wir gründlich vorsensitivierten Tauben eine niedrige $0,1 \mathrm{mg} / \mathrm{kg}$ Apodosis, die von sich aus kaum Picken hervorrief, einer Gruppe als CS $10 \mathrm{~min}$ vor einer höheren $0,75 \mathrm{mg} / \mathrm{kg}$ Apodosis als US über 5 Tage gegeben. Einer anderen Gruppe wurde zur Kontrolle dieselbe niedrige Dosis etwa 2 Stunden vor der hohen Dosis auch an 5 Tagen verabreicht. Es zeigte sich, dass sich bei der ersten Gruppe das Picken auf die niedrige Dosis Apo hin signifikant erhöhte, während bei der anderen Gruppe das Picken auf dieselbe Dosis hin durchgehend niedrig blieb. Die Verabreichung einer niedrigen Dosis Apo ist also ein wirksamer CS, der durch Kontingenz mit höherer Dosis eine CR zu verursachen vermag.

Man muss also bei Sensitivierungsversuchen mit dem Wirken eines $\mathrm{CS}_{\text {apo }}$, eine Wahrnehmung der Umgebung, die durch den Apo verursachten Zustand ,gefärbt" ist, und einem $\mathrm{CS}_{\text {noa }}$ (noa=kein Apo), einer apounbeeinflussten, normalen Wahrnehmung derselben Umgebung unterscheiden. Das erklärt natürlich, warum die CR auf dem Experimentalkäfig nach Salbehandlung schwächer als der Reaktionszuwachs bei der vorausgehenden Sensitivierung auf Apo ausfällt. Natürlich deswegen, weil bei erster Situation der ganze $\mathrm{CS}_{\text {apo }}$ wirkt und bei letzter lediglich der $\mathrm{CS}_{\text {noa }}$. Âhnlich kann die Löschungsprozedur, eine wiederholte $\mathrm{CS}_{\text {noa }}$ Präsentation, den Sensitivierungszuwachs, den $\mathrm{CR}_{\text {apo, }}$ nicht wesentlich mindern, weil sie nur den $\mathrm{CR}_{\text {noa }}$ betrifft, aber der wirk-

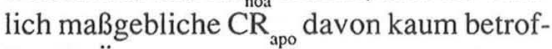
fen ist. Ähnlich dürfte die Vorabgewöhnung an den Versuchskäfig als $\mathrm{CS}_{\text {noa }}$ verlaufen, bei dem latente Hemmungsversuche sich kaum auswirken, weil ja bei der anschließenden Sensitivierung die verschiedenartige $\mathrm{CS}_{\text {apo }}$ wirksam wird. Neuerdings wird akzeptiert, dass der US auch bei üblicheren Fällen von klassischer Konditionierung häufig einen CS Nebeneffekt hat.

Zusätzliche Versuchsergebnisse belegen besonders eindrucksvoll die gelernte Natur der Sensitivierung auf Apo. Eine Gruppe Tauben wurde in einem weißwandigen grünbepunkteten Käfig auf Apo sensitiviert und parallel auch in einem schwarzwandigen, rotbepunktetem Käfig salbehandelt. Eine andere Gruppe Tauben wurde genau umgekehrt trainiert. Anschließend wurden die Tauben beider Gruppen sowohl unter Apo als auch unter Sal in einen Wahlkäfig, 
der schachbrettartig mit beiden Arten von Wanddekor versehen war, geprüft. Sie bepickten hier unter beiden Bedingungen fast ausschließlich die mit der vorherigen Aposensitivierung verbundenen Punktfelder (Abbildung $3 \mathrm{~A}$ ). Als beide Taubengruppen ein Umkehrungstraining erfuhren, also unter Apoeinfluss in den Käfig gesetzt wurden, in dem sie ursprünglich unter Sal gekommen waren, und umgekehrt nach Sal in den Käfig, den sie vormals unter Apo erfahren hatten, zeigten erneute Schachbrettkäfigtests, dass die Tauben umdressiert waren und die zuletzt apokontingenten Reizfelder wählten.

Ein Modell dafür, wie die konditionierungsbedingte Sensitivierung auf neuronalen Substraten ablaufen könnte, zeigt Abbildung 3B. Es fußt auf einem Vorschlag, den Wickens 1990 zum Lernen von neuen senso-motorischen Verknüpfungen gemacht hat. Mittelpunkt ist ein Neuron, genauer natürlich eine Population solcher Neurone, das im ventralen Striatum liegen dürfte und welches, wenn es von dem US Apo postsynaptisch aktiviert wird, mittelbar zu UR Picken führt. Dieses Neuron wird von der bereits erwähnten mes-telenzephalen Bahn daerg innerviert. Darüber hinaus projiziert auch eine glutamat(glu)erge kortiko-striatale Bahn (solch eine Projektion gibt es auch bei Vögeln!) auf dieses Neuron. Diese Bahn führt dem Neuron Informationen über potentielle CS zu. Es wird angenommen, dass ausgewählte Glusynapsen bei einer simultanen glu- und daergen Aktivierung des Pickneurons verstärkt werden (CS-UR Bahnung). Allerdings sind in dem afferenten CS-Weg weitere daerge Synapsen zwischengeschaltet (etwa in der Retina, Tectum; s.o.) die, wenn sie durch systemisches Apo angesprochen werden, zur o.g. Wahrnehmungsveränderung führt. Die umgebungsspezifische Aposensitivierung entspräche demnach einer Da-geförderten Langzeitpotenzierung, wie sie physiologisch/pharmakologisch in striatalen Substraten bei Säugern schon überzeugend nachgewiesen worden ist (Kelley 1999).

\section{Neuropharmakologie}

Ergebnisse zur Wirkung von Da- und Gluantagonisten stützen das Modell. Eine gleichzeitige Verabreichung von $\mathrm{Hal}(0,3$ $\mathrm{mg} / \mathrm{kg}$ i.m.) verhinderte die Entwicklung der Sensitivierung auf die Standarddosis Apo und verhinderte auch die anschließend getestete $\mathrm{CR}_{\text {noa }}$ völlig. Nach einer normalen Sensitivierung auf Apo hemmen aber Halgaben nicht die Expression des $\mathrm{CR}_{\text {nos }}$

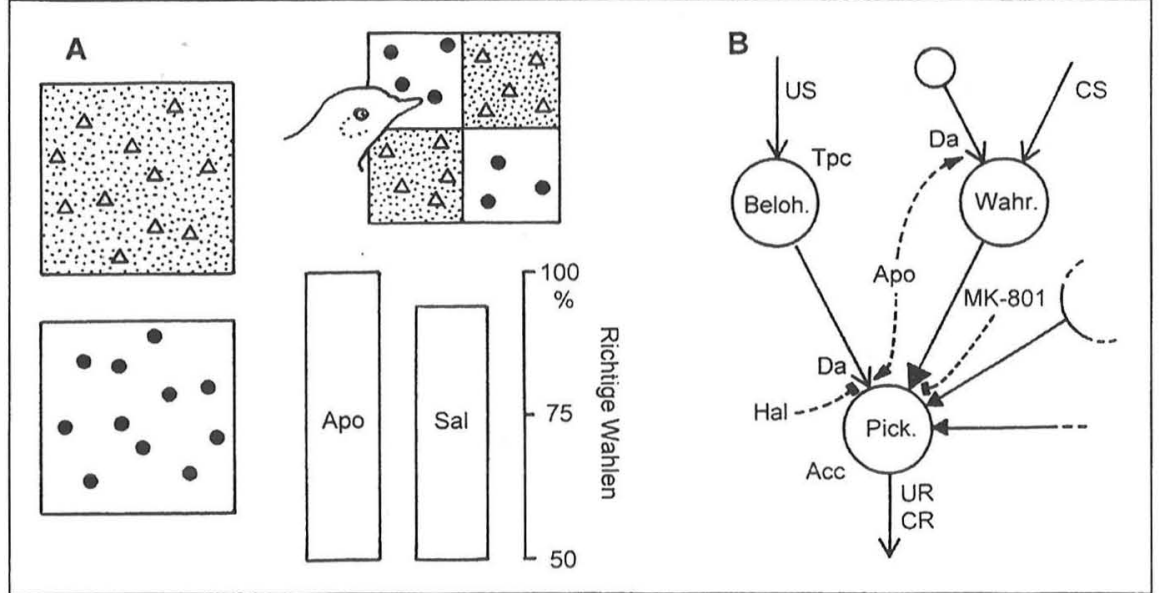

Abb. 3: A: Reizdiskrimination. B: Modell zur Aposensitivierung bei Tauben (rechts). Abkürzungen: siehe Text, Beloh.: Belohnungssystem; Tpc: nigrotegmentales Homologon der Vögel, Pick: pickmotorisches Neuron; Wahr.: Wahrnehmungsneuron. Die ausgefüllten Glusynapsen werden als konditionierungsbedingt veränderlich angenommen (verändert nach Keller et al., 2002, Delius et al., 2001).
(Abbildung 4A). Übrigens, obwohl Hal das apoverursachte Picken stark unterdrückt, hat es auffälligerweise keinerlei hemmende Wirkung auf das normale Futterpicken von Tauben. Für das Zustandekommen der Sensitivierung ist eine Aktivierung von DaRezeptoren, insbesondere D2 Rezeptoren, die Hal besonders blockiert, offensichtlich essentiell. Ist aber die Sensitivierung auf Apo vorher ungehindert etabliert worden, kann Hal den $\mathrm{CR}_{\text {noa }}$ nicht mehr unterdrükken. Ähnliche Ergebnisse lieferte der allerdings intracerebral (bilateral $3 \mu \mathrm{g} / \mu \mathrm{l}$ ) verabreichte D1 Rezeptorblocker, SCH23390 (Acerbo, in Vorb.). Kurzum, die Akquisition, aber nicht die Expression der $\mathrm{CR}_{\text {no }}$ wird von Da-Antagonisten unterdrückt.

Zum Nachweis der Beteiligung einer veränderlichen, gluergen, synaptischen Übertragung wurde der Nmda-Rezeptorantago- nist Dizilocepin (MK-801) verwandt. Nach herkömmlichen, vorwiegend aus der neurophysiologischen Langzeitpotenzierungs(LTP)-forschung abgeleiteten Vorstellungen spielen die Nmdarezeptoren bei der Akquisition einer CR eine wesentliche, anstoßende Rolle. Nicht so bei der späteren Expression der CR, wenn die Amparezeptoren die maßgebliche Rolle spielen. Tauben wurde kurz vor der Verabreichung der Standarddosis Apo mit 0,1 mg/kg MK-801 injiziert. Dies hatte eine stark unterdrückende Wirkung auf die Entwicklung des Sensitivierungszuwachses, der $\mathrm{CR}_{\text {apo }}$ : er war um gut die Hälfte reduziert. Wenn die Tauben anschließend an diese Behandlung nach einer Salgabe in den Versuchskäfig kamen, war so gut wie gar keine Pick-CR ${ }_{\text {noa }}$ mehr zu beobachten. Wenn aber die Tauben noch weiter unter Sal im Versuchskäfig getestet

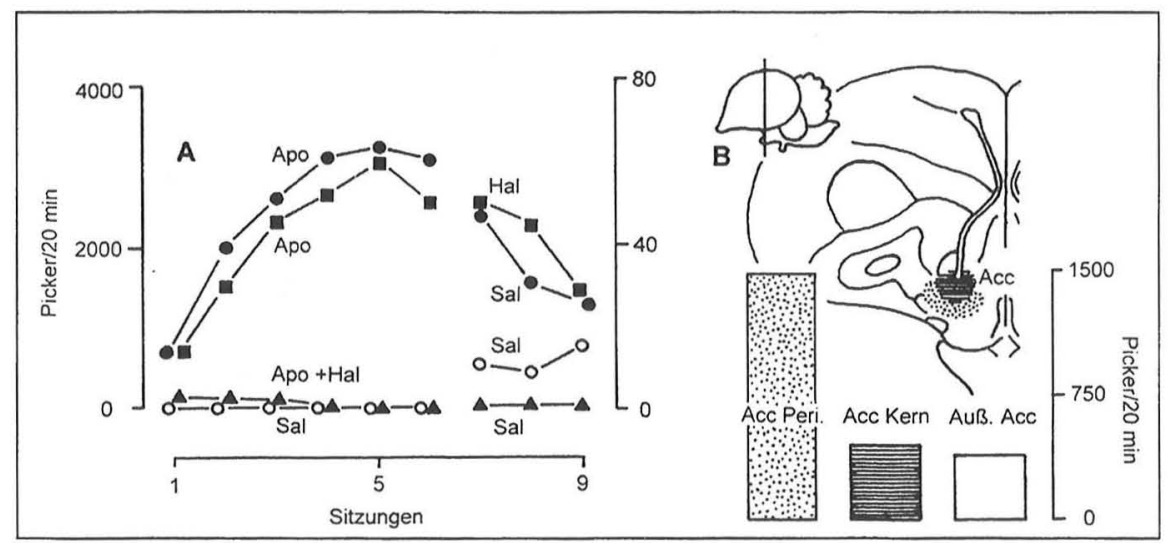

Abb. 4: A: Haloperidolwirkung auf Sensitivierung und konditionierte Reaktion. B: Apomorphinwirkung im Nucleus accumbens Kern und Peripherie (Auß. Acc; außerhalb dieses Gebietes; verändert nach Acerbo et al. 2002, Acerbo et al. 2003). 
wurden, dann trat nach einigen Tagen verzögert doch noch eine vermutliche $\mathrm{CR}_{\text {noa }}$ auf. Weiterhin zeigten vorher auf Apo sensitivierte Tauben, denen vor Tests im Versuchskäfig 0,1 mg/kg MK-801 anstatt Sal verabreicht wurde, ein deutlich vermindertes Pick $\mathrm{CR}_{\text {noa }}$. Demnach würde MK-801 nicht nur bei der Akquisition, sondern, etwas unkonventionell, auch bei der Expression des CR zwar schwächer, aber dennoch hemmend wirken. Wie das im Einzelnen zustande kommt, verstehen wir noch unzureichend, vermuten aber, dass NMDA-Rezeptoren, anders als üblich angenommen, auch nach dem Lernen beim Abruf eine gewisse Rolle spielen (s.a. Tzschentke und Schmidt 1998 und unten). Übrigens, MK801 hatte in derselben Dosis, wie zu erwarten, überhaupt keinen Effekt auf das normale Nahrungspicken (Acerbo und Delius, in Vorb.).

Zum neuronalen Substrat der konditionierten Sensitivierung haben uns, neben Wissen zur neuroanatomischen Verteilung von $\mathrm{Da}, \mathrm{Da}$-Rezeptoren, usw., besonders Gehirnautoradiographien von Tauben, die während eines Apopickanfalles mit $\left[{ }^{14} \mathrm{C}\right]-$ 2-Deoxyglucose auf lokale Stoffwechselaktivierung hin markiert wurden, Hinweise gegeben. Eine der in Frage kommenden Strukturen, den Nucleus accumbens (Acc) im ventralen Vorderhirn, haben wir näher untersucht. Bilaterale Mikroinjektionen von 20 ug/ul linksdrehendes Apo in die Peripherie (weniger im Kern) des Acc bei entsprechend kanulierten Tauben führten zu deutlichen Pickschüben (Abbildung 4B). Ebenfalls zeigte sich, dass eine vorher futterbelohnt gelernte, visuelle Reizdiskrimination durch bilaterale Injektionen von $1 \mathrm{ug} /$ ul der Glu-Nmda Antagonisten 5- oder 7aminophosphono-heptanoische Säure in den Acc selbst signifikant gestört wird. Es wäre aber verfrüht, sich auf diese Lokalisation schon festzulegen. Exploratorische Apomikroinjektionen zeigen, dass weitere Gehirnorte bei den Pickanfällen beteiligt sind. Nur einer davon, der Nucleus basalis, ein eigentümlicher, zum Trigeminussystem gehörender Kern im vorderen Vorderhirn der Sauropsiden, ist von uns näher untersucht worden; ob aber bei ihm eine gluerge Übertragung eine Rolle spielt, ist noch offen. Die o.g. Autoradiographien suggerieren weiterhin, dass beim Apopicken eine Vielzahl von Gehirnarealen involviert sind, d.h. ein weitverteiltes Netzwerk, das schätzungsweise aus rund einer Million Nervenzellen besteht. Brunelli et al. wiesen z.B. bereits 1975 nach, dass auch noch vorderhirnlose Tauben auf
Apogaben hin picken, allerdings wohl ohne eine Sensitivierung auf den Da-Agonisten zu zeigen.

\section{Ausblick}

Neben der Tatsache, dass die konditionierte Sensitivierung auf Apo bei Tauben ohne Zweifel Licht auf die sicherlich komplexere Sensitivierung bei den suchtauslösenden Psychostimulanzien wirft, dürfte es ein einfaches, preiswertes System sein, bei dem die Interaktionen von dopaminergen und glutamatergen Mechanismen beim sensomotorischen, assoziativen Lernen gründlicher untersucht werden können. Denkbar ist, dass sich das System, z.B. bei Hühnerküken, auf Hirndickschnittpräparate reduzieren lassen würde, bei denen eine detaillierte, neuro-pharmakologische Analyse möglich wäre.

\section{Danksagungen}

Wir danken Dr. Mark Cleaveland, Dr. Martina Siemann und Tech. Ass. Ines Krug für vielseitige Beiträge. Die Deutsche Forschungsgemeinschaft hat unsere Forschung langjährig unterstützt.

\section{Literatur}

Anagnostaras, S.G. und Robinson, T.E. (1996): Sensitization to the psychomotor stimulant effects of amphetamine. Modulation by associative learning. Behav. Neurosci. 110: 1397-1414.

Godoy, A. und Delius, J.D. (1999): Sensitization to apomorphine in pigeons is due to conditioning, subject to generalization but resistant to extinction. Behav. Pharmacol. 10: 367-378.

Keller, S. und Delius, J. D. (2001): Discriminative learning occasioned by the administration of a dopamine agonist. Psychopharmacol. 157: 320-323.

Keller, S., Delius, J.D. und Acerbo, J.D. (2002): Sensitization to apomorphine: evoking conditions, context dependence and effect persistence of. Behav. Pharmacol. 13: 1-14.

Kelley, A.E. (1999): Neural integrative activities of nucleus accumbens subregions in relation to learning and motivation. Psychobiol. 27: 198 213.

Eine ausführliche Literaturliste kann bei den Autoren angefordert werden.

\section{Kurzbiographien}

Juan D. Delius: geb. in Essen 1936, aufgewachsen in Argentinien 1937-1954. Studium der Biologie 1955 in Bonn, Freiburg, Göttingen und Oxford. Dissertation 1959 bei Prof. Niko Tinbergen, Oxford, Promo- tion Dr. rer. nat. 1961 in Göttingen. Assistent 1962 Zoologie, Oxford. Lecturer 1967 Psychologie, Durham (UK). Wiss. Rat und Prof. Psychologie, 1974 Bochum, Prof. 1987 Psychologie, Konstanz, a.D. 2001, Konstanz. Gastforscher in San Diego (USA), Frankfurt, Seewiesen, Iztacala (Mex.), Paris, Buenos Aires, Durham und Barcelona. Forschungsgebiet: Vergleichende und neurobiologische Psychologie.

Martin J. Acerbo: geb. in Arrecifes (Arg.) 1969. Studium der Biologie, Universität Buenos Aires 1988, Lic. Biol. 1996, Promotion Dr. rer. nat. bei J.D.D., Konstanz 2001. Gastaufenthalte Biopsychologie, Bochum und animalische Biochemie und Genetik, Ivanka p. D., Slovakien. 2002 Postdok., Biopsychologie (Prof. Terry Robinson), Universität Michigan. Forschungsgebiet: Vehaltens- und Molekularpharmakologie.

Sabine Keller: geb. 1963 in Simmerath. Studium der Agrarwissenschaften 1985Universität Bonn Dipl. ing. agr. 1991, Studium der Veterinärmedizin 1994 Universität München, Erst. Staatsex. 1997, Promotion Dr. rer. nat. bei J.D.D., Konstanz 2001. Freiberuflich tätig in Konstanz.

Adriana Grodoy: geb. in Buenos Aires 1969. Studium der Biologie 1988, Universität Buenos Aires, Lic. Biol. 1995. DAAD Stipendiantin in Konstanz, Promotion Dr. rer. nat. bei J.D.D., Konstanz 2000. Universitätspreis 2001. Freiberuflich tätig in Málaga.

\section{Korrespondenzadresse}

Prof. Dr. Juan D. Delius

Allgemeine Psychologie

Universität Konstanz

D-78457 Konstanz

Email: juan.delius@uni-konstanz.de 